\title{
Trichinella spiralis shares epitopes with human autoantigens
}

\author{
Ivana Radovic ${ }^{1 /+}$, Alisa Gruden-Movsesijan'1, Natasa Ilic ${ }^{1}$, \\ Marija Mostarica-Stojkovic ${ }^{2}$, Ljiljana Sofronic-Milosavljevic ${ }^{1}$ \\ 1Reference Laboratory for Trichinellosis, Institute for the Application of Nuclear Energy \\ 2Faculty of Medicine, Institute of Microbiology and Immunology, University of Belgrade, Belgrade, Serbia
}

\begin{abstract}
Like other helminths, Trichinella spiralis has evolved strategies to allow it to survive in the host organism, including the expression of epitopes similar to those present in either expressed or hidden host antigens. To identify $\mathrm{T}$. spiralis-derived antigens that are evolutionarily conserved in the parasite and its host and that could be responsible for its evasion of the host immune response, we examined the reactivity of six different types of autoantibodies to T. spiralis larvae from muscle. T. spiralis antigens that share epitopes with human autoantigens were identified by assessing the cross-reactivity of autoantibody-containing serum samples with $\mathrm{T}$. spiralis antigens in the absence of specific anti-parasite antibodies. Of the 55 autoantibody-containing human serum samples that we analysed by immunohistological screening, 24 (43.6\%) recognised T. spiralis muscle larvae structures such as the subcuticular region, the genital primordium or the midgut. Using Western blots, we demonstrated that the same sera reacted with 24 protein components of $\mathrm{T}$. spiralis muscle larvae excretory-secretory L1 antigens. We found that the human autoantibodies predominantly bound antigens belonging to the TSL1 group; more specifically, the autoantibody-containing sera reacted most frequently with the 53-kDa component. Thus, this protein is a good candidate for further studies of the mechanisms of T. spiralis-mediated immunomodulation.
\end{abstract}

Key words: human autoantibodies - Trichinella spiralis antigens - conserved epitopes

The immune system, which evolved to detect, inactivate and/or destroy invaders, includes both innate and adaptive responses that are activated upon encounter of foreign epitopes. However, the immune system must also be tolerant and able to distinguish between self and non-self antigens. The consequence of a breakdown in self-tolerance is autoimmunity. In autoimmune responses, the immune system attacks self organs and tissues as if they were intruders. Autoimmune diseases arise from combinations of genetic, environmental and immunological factors (Christen \& von Herrath 2005). Among the environmental factors that are thought to influence autoimmunity, infections with various pathogens are prime candidates to initiate or exacerbate autoimmune diseases. Several mechanisms have been proposed by which pathogens might cause autoimmunity, such as cross-reactivity between microbial and self determinants, bystander activation or expression of altered self determinants arising from the tissue damage caused by a strong inflammatory response during infection (Ercolini \& Miller 2009). In contrast, many epidemiological and experimental studies of animal models of human autoimmune diseases have shown that infections with parasites, and in particular with helminths, might prevent rather than induce or accelerate autoimmune and

Financial support: Ministry of Education and Science, Republic of Serbia (173047)

+ Corresponding author: ivanar@inep.co.rs

Received 13 September 2011

Accepted 16 February 2012 allergic diseases (Cooke et al. 2004, Wilson \& Maizels 2004). Chronic helminth infections are accompanied by a wide range of immunomodulatory effects (Maizels \& Yazdanbakhsk 2003) that enable the survival of both the parasite and the host organism. The parasitic nematode Trichinella spiralis invades striated muscle cells and transforms them into nurse cells surrounded by a collagen capsule (Despommier 1998, Wu et al. 2008). From this immunoprivileged site, muscle larvae produce excretory-secretory products (ES L1) that manipulate the host immune response to other, irrelevant antigens and limit the inflammatory response (Fabre et al. 2009). Infection with $T$. spiralis (Khan et al. 2002, Saunders et al. 2007, Gruden-Movsesijan et al. 2010) or treatment with a $T$. spiralis antigen preparation (Motomura et al. 2009) may prevent the development of several autoimmune diseases, such as type 1 diabetes, experimental autoimmune encephalomyelitis (EAE) and experimental colitis, by skewing the immune response towards a Th2 or regulatory profile. Studies of the mechanisms underlying the amelioration of autoimmunity that is observed during $T$. spiralis infection have focused on both the immune system and parasite molecules involved in immune modulation. As it is clear that the administration of live T. spiralis larvae carries potential risks, it is necessary to isolate and characterise the active components of the T. spiralis organism. The identification and characterisation of the ES L1 products that are involved in shaping the host immune response are the focus of current investigations (Guiliano et al. 2009). Gong et al. (2011) have recently shown that tumour-associated antigens exist in T. spiralis and are important for the antitumour effects of $T$. spiralis infection. The validation of the diagnostic tools for the detection of anti-T. spiralis 
antibodies has indicated that antibodies from the sera of patients with various autoimmune disorders, but without Trichinella infection can bind T. spiralis antigens (Robert et al. 1996, Gomez-Morales et al. 2008). We hypothesise that this cross-reactivity could result from the presence of common epitopes in the parasitic antigens and the autoantigens that may be involved in modulating the immune response. In the present study, we used immunohistological staining and Western blots to detect T. spiralis antigens that cross-react with autoantibodies. These data allow us to identify the structures that we should focus on in future investigations of the recently described $T$. spiralis-mediated amelioration of EAE (Gruden-Movsesijan et al. 2008, 2010). We found that a large proportion of autoantibody-containing human sera recognized $T$. spiralis antigens.

\section{MATERIALS AND METHODS}

Serum samples - Serum samples $(\mathrm{n}=55)$ were obtained from two sources: the Clinic of Alergology and Immunology, Clinical Centre of Serbia, Belgrade and Institute for the Application of Nuclear Energy (INEP) (ISO/IEC 17025:2006). The sera used in this study were identified as positive for high titres of autoantibodies using commercial diagnostic kits [indirect immunofluorescence (IIF) kits and Avitex RF, Omega Diagnostics, Hillfoots Business Village, Alva, Scotland, UK]. Twenty serum samples contained anti-nuclear antibodies (ANAs), 10 contained anti-mitochondrial antibodies (AMAs), eight contained anti-smooth muscle antibodies (ASMAs), four contained anti-keratin antibodies (AKAs), seven contained anti-cyclic citrullinated protein (anti-CCP) antibodies and six contained rheumatoid factor (RF). All of the sera were examined for the presence of anti-T. spiralis antibodies or T. spiralis antigens through specific in-house testing at the Serbian Reference Laboratory for Trichinellosis at INEP.

These tests, which included ELISAs specific for anti-Trichinella antibodies, IIF of whole larvae sections, Western blots with antibodies specific for ES L1 antigens and dot blots using antibodies specific for ES Ag were used to confirm that the patients from whom sera were collected were not infected with $T$. spiralis.

For the positive and negative controls, we used the monoclonal antibody (MoAb) 7C2C5, which recognises an epitope on the TSL1 group of antigens unique to the Trichinella genus (Gamble \& Graham 1984), serum with a known titre of anti-Trichinella antibodies from a patient infected with $T$. spiralis and serum with no parasite-specific antibodies or autoantibodies. We chose the dilution of serum to use for the positive control by testing serial dilutions $(1: 25,1: 50,1: 100,1: 200,1: 400)$ of the Trichinella-positive control in Western blots specific for ES L1 antigens. We found that a dilution of 1:50 allowed us to distinguish the Trichinella-specific triad of antigens at $45 \mathrm{kDa}, 49 \mathrm{kDa}$ and $53 \mathrm{kDa}$ and thus to clearly discriminate between Trichinella-positive and Trichinella-negative sera. The serum samples containing autoantibodies were examined at the same dilution using the same test.
Parasite - T. spiralis L1 larvae were recovered from infected Wistar rats by digesting the carcasses in prewarmed gastric juice $(1 \%$ pepsin in $1 \% \mathrm{HCl}, \mathrm{pH} 1.6-1.8)$ (Gamble et al. 2000). After stirring the digested samples for $4 \mathrm{~h}$ at $37^{\circ} \mathrm{C}$, the larvae were allowed to sediment. The pepsin- $\mathrm{HCl}$ solution was removed by aspiration and the L1 larvae were washed with saline. The larvae were collected and used for either the preparation of slides for IIF assays or the production of ES L1 antigens.

ES L1 antigen preparation - Muscle larvae were kept under controlled conditions $\left(37^{\circ} \mathrm{C}, 10 \% \mathrm{CO}_{2}\right)$ in complete Dulbecco's modified Eagle's medium (Sigma-Aldrich, Gmbh, Taufkirchen, Germany) supplemented with 10 mM HEPES, $2 \mathrm{mM}$ L-glutamine, $1 \mathrm{mM}$ Na pyruvate (all from Sigma-Aldrich, Gmbh, Taufkirchen, Germany) and $50 \mathrm{U} / \mathrm{mL}$ Pen/Strep (Galenika, Belgrade, Serbia) for $18 \mathrm{~h}$. The ES L1 products of the muscle larvae were dialysed from the culture supernatants, concentrated and stored until use at $-20^{\circ} \mathrm{C}$.

IIF for detection of reactivity with T. spiralis muscle larvae - The slides for IIF were prepared from T. spiralis muscle larvae fixed in cold ethanol and xylene and embedded in paraffin for histological analysis. Five-micron sections were deparaffinised in xylene, rehydrated with a graded alcohol series, washed and incubated with human sera that had been diluted 1:40 in phosphate buffered saline (PBS). The sections were then washed, incubated with sheep anti-human immunoglobulin conjugated to fluorescein isothiocyanate (FITC Trichinella spiralis Antibody Detection Kit, INEP, Belgrade, Serbia) and examined by ultraviolet microscopy (AXIO Imager A1, Carl Zeiss AG, Gottingen, Germany).

Immunohistochemical staining of T. spiralis muscle larvae using peroxidase-labelled anti-human Ig - The slides for immunohistochemical staining were prepared as described above. The endogenous peroxidase activity was quenched with $3 \%$ hydrogen peroxide in methanol for $20 \mathrm{~min}$ at room temperature (RT). The sections were then incubated for $30 \mathrm{~min}$ with human sera diluted 1:40 in PBS, washed three times in PBS and incubated with sheep anti-human $\operatorname{IgG}$ antibody conjugated with horseradish peroxidase (HRPO) (INEP, Belgrade, Serbia) for $30 \mathrm{~min}$. The reaction was developed by adding the chromogenic substrate $0.05 \% \quad 3.3$ '-diaminobenzidine tetrahydrochloride (DAB) (Vector Laboratories, Burlingame, CA, USA) according to the manufacturer's instructions. For contrast, all of the sections were stained with haematoxylin, dehydrated, cleared in xylene and mounted in Canada balsam (Sigma-Aldrich, Gmbh, Taufkirchen, Germany). The stained sections were viewed using light microscopy (AXIO Imager A1, Carl Zeiss AG, Gottingen, Germany).

Western blot analyses of T. spiralis ES L1 products The protein components of the ES antigens of the T. spiralis muscle larvae were separated by electrophoresis under reducing conditions using 5-20\% gradient polyacrylamide gels. An immunoblot assay was utilised to identify the immunoreactive components of the ES antigens that were recognised by autoantibodies present in the human sera. After electrophoresis, the protein components were 
transferred to a polyvinylidene difluoride membrane. A mixture of proteins, which included lysozyme (14.4 kDa), soybean trypsin inhibitor $(21.5 \mathrm{kDa})$, carbonic anhydrase (31 kDa), ovalbumin $(45 \mathrm{kDa})$, bovine serum albumin (BSA) $(66.2 \mathrm{kDa})$ and phosphorylase b $(97.4 \mathrm{kDa})$ was used as a molecular weight standard (Bio-Rad Laboratories, USA). The membranes were blocked with 1\% BSA in TBS (50 mM Tris-HCl, $150 \mathrm{mM} \mathrm{NaCl}, \mathrm{pH}$ 7.6) for $1 \mathrm{~h}$ at RT. After blocking, the membranes were cut into strips and incubated with the positive control serum, the negative control serum or a 1:50 dilution of the human serum samples for $48 \mathrm{~h}$. As an additional control, we used the 7C2C5 MoAb, which identifies the specific T. spiralis epitope present in the TSL1 group of antigens (45 kDa, $49 \mathrm{kDa}, 53 \mathrm{kDa}$ ) (Gamble \& Graham 1984). The protein fractions recognised by the human sera were detected by incubating the membranes for $2.5 \mathrm{~h}$ with a 1:1.000 dilution of a sheep anti-human immunoglobulin-peroxidase conjugate (INEP, Belgrade, Serbia). The bands recognised by the MoAb 7C2C5 were detected using a 1:500 dilution of a rabbit anti-mouse immunoglobulin-peroxidase conjugate (DAKO, Hamburg, Gmbh). The blots were developed with a $0.05 \%$ solution of DAB (Peroxidase Substrate Kit-DAB, Vecor, UK).

\section{RESULTS}

Immunohistological screening of sera with autoantibodies - IIF staining of preparations of $T$. spiralis muscle larvae is a sensitive assay that is routinely used to detect anti-T. spiralis antibodies in human sera. The T. spiralis-positive serum (positive control) produced a bright, apple-green signal on the cuticle and in the stichosome (Fig. 1A), whereas the sample stained with the negative control serum did not emit fluorescence (Fig. 1B). Although the various sera containing autoantibodies did not produce the typical staining pattern that is observed when using specific anti-T. spiralis antibodies, eight $(14.5 \%)$ of the 55 sera tested produced dull IIF staining of parasite organelles. The autoantibodies stained internal structures, but not the cuticle surface (Fig. 1C-E).

Muscle sections containing larvae were examined by immunohistochemical staining with an anti-human Ig-HRPO antibody, which allowed better visualisation of the structures recognised by the autoantibodies (Fig. 2). We observed that more serum samples reacted with the parasite components using this test than using the IIF assay. Using the immunohistochemistry assay, 24 (43.6\%) of the 55 samples containing ANAs, AMAs, ASMAs, AKAs, RF or anti-CCP autoantibodies recognised structures of $T$. spiralis muscle larvae other than the stichosome, such as the subcuticular region, the genital primordium or the midgut (Fig. $2 \mathrm{C}, \mathrm{D}, \mathrm{E}$ ).

Western blot analyses of autoantibody reactivity with ES L1 - We used Western blot analyses to confirm the results that we obtained using immunohistochemistry and found that the same $24(43.6 \%)$ of 55 autoantibodycontaining sera recognised ES L1 antigen. The MoAb 7C2C5 recognises an epitope unique to the Trichinella genus and bound a triad of bands at $45 \mathrm{kDa}, 49 \mathrm{kDa}$ and $53 \mathrm{kDa}$ (Fig. 3, Lane A). This profile is characteristic of T. spiralis-positive samples (Fig. 3, Lane B). The detection of the T. spiralis-specific triad proves the presence of specific anti-Trichinella antibodies and confirms that the sample is from an individual infected with this helminth. Apart from the specific triad, an immunoblot analysis of T. spiralis ES antigens in the positive control serum revealed bands at $20 \mathrm{kDa}, 25 \mathrm{kDa}, 27 \mathrm{kDa}, 32$ kDa, $59 \mathrm{kDa}, 68 \mathrm{kDa}$ and $130 \mathrm{kDa}$ (Fig. 3, Lane B). The negative control serum did not react with $T$. spiralis ES L1 antigens in the Western blot (Fig. 3, Lane C).
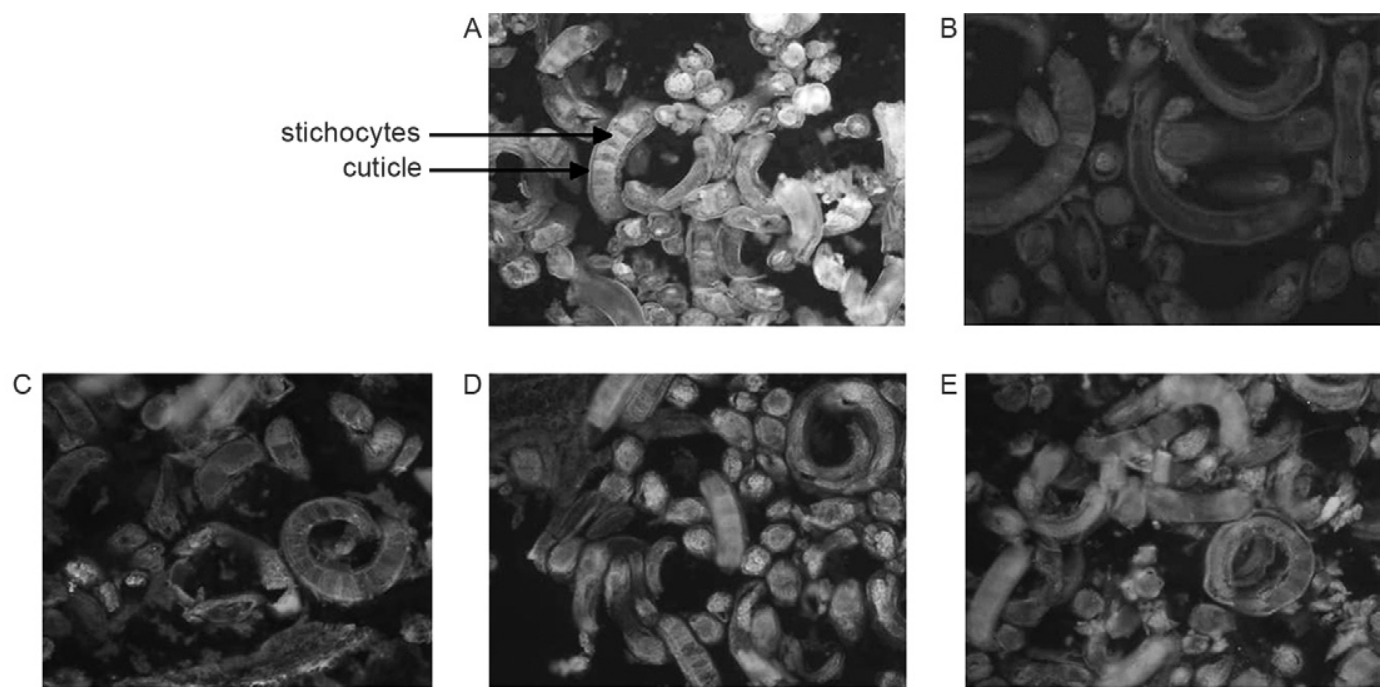

Fig. 1: human autoantibodies cross react with Trichinella spiralis: indirect immunofluorescence (IIF) staining. T. spiralis positive control serum (A), T. spiralis negative control serum (B), serum samples positive for anti-nuclear antibody (C), anti-mitochondrial antibody (D) and anti-keratin antibody (E) in dilution 1:40. Example that autoantibodies provided different IIF staining compared to anti-parasite antibodies. A-E: 20X magnification. 

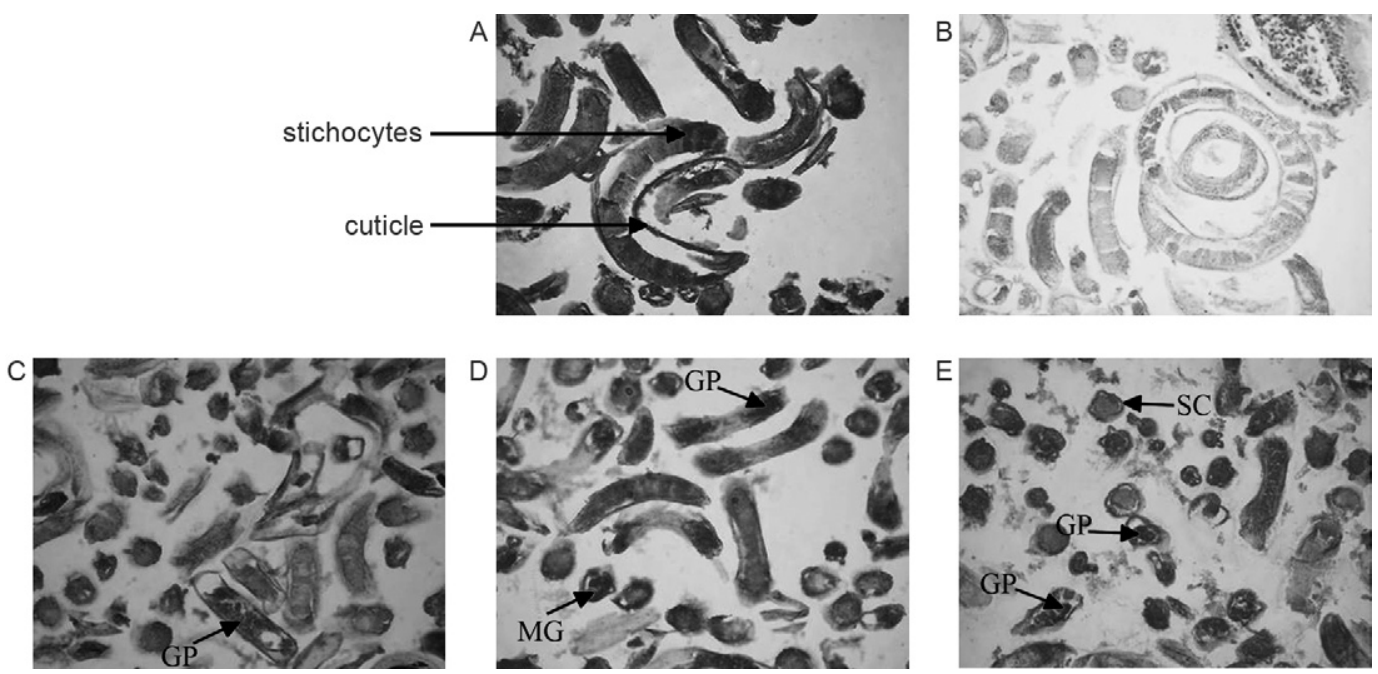

Fig. 2: human autoantibodies cross react with Trichinella spiralis. Immunohistochemical staining using anti-Ig-HRPO as a secondary antibody. T. spiralis positive control serum (A), T. spiralis negative control serum (B); serum samples positive for anti-nuclear antibody (C), antimitochondrial antibody (D) and anti-smooth muscle antibody (E) all in dilution 1:40. Example that autoantibodies stained in the zone of genital primordium (GP), midgut (MG), subcuticular (SC) layer of muscle larvae. A-E: 20X magnification.

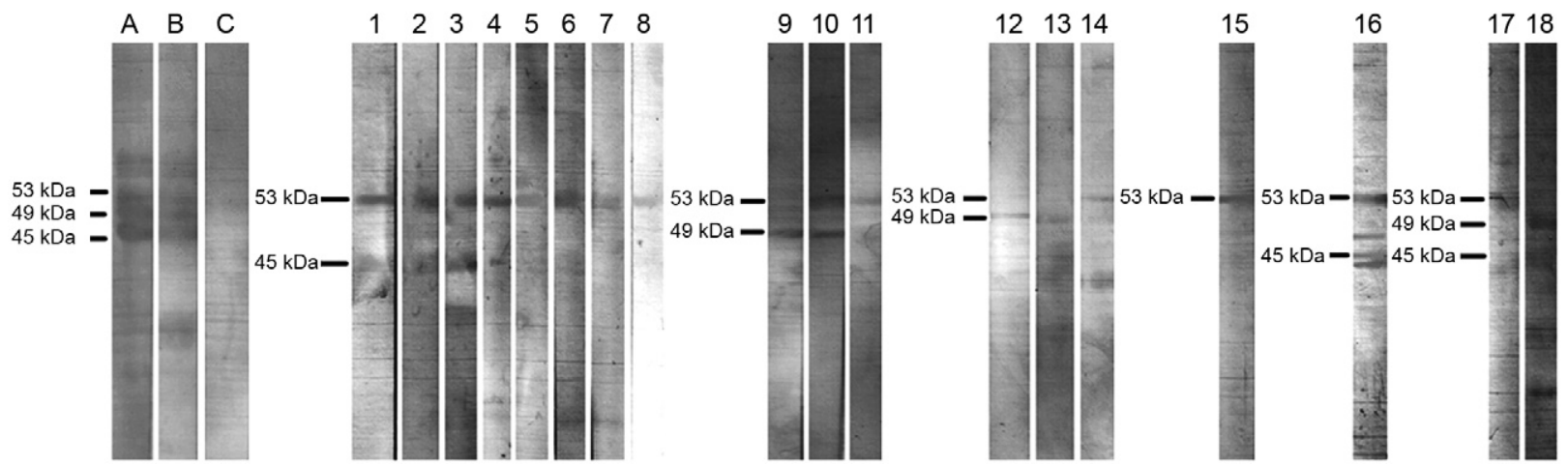

Fig. 3: Western blot analyses of sera with autoantibodies to excretory-secretory L1 antigen - 7C2C5 monoclonal antibody. Lane A: Trichinella spiralis specific triad (45 kDa, $49 \mathrm{kDa}, 53 \mathrm{kDa})$; B: T. spiralis positive control; C: T. spiralis negative control; 1-18: serum samples with autoantibodies that stain one or two bands from the Trichinella specific triad ( $45 \mathrm{kDa}, 49 \mathrm{kDa}, 53 \mathrm{kDa})$; 1-8: sera samples positive for anti-nuclear antibody; 9-11: sera samples positive for anti-mitochondrial antibody; 12-14: sera samples positive for anti-smooth muscle antibody; 15: serum sample positive for anti-keratin antibody; 16: serum sample positive for anti-cyclic citrullinated protein antibody; 17-18: sera samples with increased level of rheumatoid factor.

Most of the autoantibody-containing sera reacted weakly and produced a variable banding pattern. The molecular masses of the 24 recognised proteins varied greatly, ranging from $15-130 \mathrm{kDa}$. However, none of the examined sera bound all three components of the T. spiralis-specific triad. Of the 24 sera that were found to be T. spiralis-reactive by Western blot, 18 (75\%) recognised one or two bands of the T. spiralis-specific triad (indicated bands) (Fig. 3, Lanes 1-18). Fourteen of these sera $(58.3 \%)$ reacted with the $53-\mathrm{kDa}$ band. The autoantibody-containing sera also reacted with $43-\mathrm{kDa}$ (10/24; 41.7\%), 45-kDa (6/24; 25\%) and 49-kDa (6/24; $25 \%$ ) bands belonging to the TSL1 group. The sera we tested also recognised high molecular mass bands of 115 $(5 / 24 ; 20.8 \%)$ and $130 \mathrm{kDa}(8 / 24 ; 33.3 \%)$, as well as low molecular mass bands of $16 \mathrm{kDa}, 20 \mathrm{kDa}, 23 \mathrm{kDa}, 25$ $\mathrm{kDa}, 27 \mathrm{kDa}, 32 \mathrm{kDa}, 35 \mathrm{kDa}$ and $36 \mathrm{kDa}$. The sizes of the bands that reacted with the different serum samples are provided in Table.

\section{DISCUSSION}

In the current study, we have demonstrated that human autoantibodies from sera without either T. spiralisspecific antibodies or antigens recognise some parasite antigens. We observed that a number of autoantibodycontaining sera reacted with the sections containing $T$. spiralis larvae in IIF assays and in immunohistochemical assays using anti-human Ig-HRPO. However, the pattern of fluorescence or peroxidase staining was clearly different from the pattern obtained using serum that was 
TABLE

Distribution of antigenic bands for the individual autoantibodies revealed in the Western blot with excretory-secretory antigen for serum samples that stain one or two bands from the Trichinella specific triad

\begin{tabular}{|c|c|c|c|c|c|c|c|c|c|c|c|c|c|c|c|c|c|c|c|}
\hline \multirow[b]{2}{*}{ Sample source } & \multirow[b]{2}{*}{ Tested } & \multicolumn{18}{|c|}{ Number of samples recognizing band (kDa) } \\
\hline & & 14 & 16 & 20 & $22-25$ & 27 & 32 & $35-36$ & $38-39$ & 40 & 43 & 45 & 49 & 53 & 58 & 64 & $80-110$ & 115 & 130 \\
\hline Negative control & 1 & - & - & - & - & - & - & - & - & - & - & - & - & - & - & - & - & - & - \\
\hline Positive control & 1 & - & - & 1 & 1 & 1 & 1 & - & - & - & - & 1 & 1 & 1 & - & - & - & - & - \\
\hline 7C2C5 MoAb & 1 & - & - & - & - & - & - & - & - & - & - & 1 & 1 & 1 & - & - & - & - & - \\
\hline ANA & 20 & 2 & 2 & 3 & 4 & - & - & 1 & 3 & - & 4 & 4 & - & 8 & 1 & 1 & - & 4 & 5 \\
\hline AMA & 10 & - & - & - & - & - & - & - & - & 1 & 3 & - & 2 & 2 & - & - & 1 & - & - \\
\hline ASMA & 8 & - & - & - & - & - & 1 & 2 & 1 & - & 1 & - & 3 & 1 & - & - & 1 & - & 1 \\
\hline AKA & 4 & - & - & - & - & - & - & - & - & - & - & - & - & 1 & - & - & - & 1 & 1 \\
\hline $\mathrm{ACCP}$ & 7 & - & - & - & - & - & - & - & - & - & 1 & 1 & - & 1 & - & - & - & - & - \\
\hline $\mathrm{RF}$ & 6 & - & - & 1 & 1 & 1 & - & - & - & - & 1 & 1 & 1 & 1 & - & - & - & - & 1 \\
\hline Total & 58 & 2 & 2 & 4 & 5 & 1 & 1 & 3 & 4 & 1 & 10 & 6 & 6 & 14 & 1 & 1 & 2 & 5 & 8 \\
\hline
\end{tabular}

TSL1 group of antigens are shown in bold. ACCP: anti-cyclic citrullinated protein; AKA: anti-keratin antibody; AMA: antimitochondrial antibody; ANA: anti-nuclear antibody; ASMA: anti-smooth muscle antibody; MoAb: monoclonal antibody; RF: rheumatoid factor.

known to contain anti-T. spiralis antibodies. In support of these results, Robert et al. (1996) also observed that different autoantibodies can exhibit cross-reactivity in IIF assays. More of the serum samples reacted with the parasite components using immunohistochemical staining of muscle larvae with anti-human Ig-HRPO than using the IIF assay. This finding may be due to the ability of the former technique to provide better visualisation of the somatic muscle larvae components that reacted with autoantibodies. We performed Western blots for T. spiralis ES L1 antigens to determine which parasitic components were responsible for the autoantibody cross-reactivity observed using the IIF and immunohistochemical assays. Both the positive control serum, which contained specific anti-Trichinella antibodies, and the MoAb 7C2C5 recognised bands of $45 \mathrm{kDa}$, $49 \mathrm{kDa}$ and $53 \mathrm{kDa}$ (the Trichinella-specific triad) that contain the immunodominant epitope for the muscle larvae stage of the Trichinella genus (Gamble \& Graham 1984). The appearance of all three bands in the Western blot indicates the presence of anti-T. spiralis antibodies and confirms infection with T. spiralis. Western blot analyses of the reactivity of different autoantibodycontaining sera with these antigens revealed that the majority of autoantibodies reacted with one or two of the bands in this triad. The banding pattern observed in Western blots performed with the autoantibody-containing sera differed from the pattern obtained using the $T$. spiralis-positive sera in both the number of visualised bands and their apparent molecular mass. Some of the bands detected in the western blots performed using the autoantibody-containing sera did not appear in the blots performed using the T. spiralis-positive control. This finding is not surprising because not all of the proteins in the ES L1 mixture are immunogenic or will induce the synthesis of $T$. spiralis-specific antibodies. However, the ES L1 proteins could be recognised by other antibodies present in the examined human sera. The assays used in this study did not allow us to determine whether a band resulted from reactivity with an autoantibody or a non-self-reactive antibody. However, we can speculate that while T. spiralis were evolving alongside their hosts, they developed epitopes similar to autoantigens.

Most of the antigens recognised by the sera from patients infected with $T$. spiralis were in the range of 40$70 \mathrm{kDa}$ in size and belonged to the TSL1 group of antigens. TSL1 antigens originate from the muscle larvae stichosome and are present on the surface of the larvae and in their ES products. It has been suggested that TSL1 antigens contribute to parasitism by enabling the survival of the parasite within its host (Nagano et al. 2009). These antigens are released from the encysted muscle larvae (L1) and they continuously stimulate the host immune response (Bruschi 2002). The 45-kDa, 49-kDa and 53-kDa glycoproteins that form the Trichinella-specific triad are tyvelose-bearing antigens that are important during the intestinal phase of the infection (Wisnewski et al. 1993, Arasu et al. 1994, Romarís et al. 2002). These glycoproteins are also responsible for inducing host immunity, as they induce a powerful antibody response against tyvelose that results in the expulsion of the parasite and the protection of the host from reinfection (Appleton et al. 1991).

In this study, we found that the glycoproteins that make up the TSL1 group were recognised by a majority of the autoantibody-containing sera, indicating that cross-reactivity with Trichinella antigens is not specific to autoantibodies that recognise a certain antigen. Previous reports have noted that autoantibodies react with 
antigens from the TSL1 group, but identified targets of different molecular masses (Robert et al. 1996). The recognised proteins in the TSL1 group could be critical for parasite survival and may have been conserved during evolution. The $53-\mathrm{kDa}$ protein, which is thought to contribute to parasitism and to modulate the host immune response (Nagano et al. 2009), reacted with $58.3 \%$ of the tested autoantibody-containing sera. The other two components of the Trichinella-specific triad, the 49-kDa protein (and its $43-\mathrm{kDa}$ isoform) and the $45-\mathrm{kDa}$ protein, reacted with $41.7 \%$ and $25 \%$ of autoantibody-positive serum samples, respectively. It has been suggested that the $49-\mathrm{kDa}$ glycoprotein is involved in nurse cell formation (Wu et al. 2002), whereas the $45-\mathrm{kDa}$ antigen inhibits human neutrophil functions such as random mobility, chemotaxis, oxidative metabolism and integrin upregulation (Bruschi et al. 2000).

The cross-reactivity that has been observed between autoantibody-containing human sera and T. spiralis antigens may indicate the existence of evolutionarily conserved common epitopes among humans and parasites. It is not surprising that antibodies that recognise evolutionarily conserved epitopes have the potential to bind to structures present in different organisms and species. The ability of these antibodies to bind both human and Trichinella antigens not only provides information about the existence of shared epitopes, but also suggests that their conservation during evolution means that these structures are vital for these organisms. In addition to their above-mentioned biological functions, the parasite components recognised by the autoantibodies may be responsible for the parasite-induced immunomodulation that prevents the induction or ameliorates the course of various autoimmune diseases (Maizels et al. 2004). Future investigations will focus on the role of the 53$\mathrm{kDa}$ TSL1 antigen, which reacted most frequently with autoantibody-containing sera, in provoking tolerogenic and anti-inflammatory effects similar to those induced by $T$. spiralis infection. The identification of immunomodulatory parasite molecules and the dissection of the mechanisms that shape the host immune system should increase our understanding of the nature of helminth immune modulation and lead to novel approaches for the treatment of multiple immunological disorders.

\section{REFERENCES}

Appleton JA, Bell GR, Homan W, van Knapen F 1991. Concensus on Trichinella spiralis antigens and antibodies. Parasitol Today 7: 190-192.

Arasu P, Ellis LA, Iglesias R, Ubeira FM, Appleton JA 1994. Molecular analysis of antigens targeted by protective antibodies in rapid expulsion of Trichinella spiralis. Mol Biochem Parasitol 65: 201-211.

Bruschi F 2002. The immune response to the parasitic nemathode Trichinella and the ways to escape it. From experimental studies to implications for human infection. Curr Drug Targets Immune Endocr Matabol Disord 2: 269-280.

Bruschi F, Carulli G, Azzarà A, Homan W, Minnucci S, RizzutiGullaci A, Sbrana S, Angiolini C 2000. Inhibitory effects of human neutrophil functions by the 45-kd glycoprotein derived from the parasitic nematode Trichinella spiralis. Int Arch Allergy Immunol 122: 58-65.
Christen U, von Herrath MG 2005. Infection and autoimmunity: good or bad? J Immunol 174: 7481-7486.

Cooke A, Zaccone P, Raine T, Phillips JM, Dunne DW 2004. Infection and autoimmunity: are we winning the war, only to lose the peace? Trends Parasitol 20: 316-321.

Despommier DD 1998. How does Trichinella spiralis make itself at home? Parasitol Today 14: 318-323.

Ercolini AM, Miller SD 2009. The role of infections in autoimmune disease. Clin Exp Immunol 155: 1-15.

Fabre MV, Beiting DP, Bliss SK, Appleton JA 2009. Immunity to Trichinella spiralis muscle infection. Vet Parasitol 159: 245-248.

Gamble HR, Bessonov AS, Cuperlovic K, Gajadhar AA, van Knapen F, Noeckler K, Schenone H, Zhu X 2000. International commission on trichinellosis: recommendations on methods for the control of Trichinella in domestic and wild animals intended for human consumption. Vet Parasitol 93: 393-408.

Gamble HR, Graham CE 1984. Monoclonal antibody-purified antigen for the immunodiagnosis of trichinosis. Am J Vet Res 45: 67-74.

Gomez-Morales MA, Ludovisi A, Amati M, Cherchi S, Pezzoti P, Pozio E 2008. Validation of an enzyme-linked immunosorbent assay for diagnosis of human trichinellosis. Clin Vaccine Immunol 15: 1723-1729.

Gong P, Zhang J, Cao L, Nan Z, Li J, Yang L, Fang H, Jiao H, Jiang T, Su L, Zhang X 2011. Identification and characterization of myeloma-associated antigens in Trichinella spiralis. Exp Parasitol 127: 784-788.

Gruden-Movsesijan A, Ilic N, Mostarica-Stojkovic M, Stosic-Grujicic S, Milic M, Sofronic-Milosavljevic Lj 2008. Trichinella spiralis: modulation of experimental autoimmune encephalomyelitis in DA rats. Exp Parasitol 118: 641-647.

Gruden-Movsesijan A, Ilic N, Mostarica-Stojkovic M, Stosic-Grujicic S, Milic M, Sofronic-Milosavljevic Lj 2010. Mechanisms of modulation of experimental autoimmune encephalomyelitis by chronic Trichinella spiralis infection in Dark Agouti rats. Parasite Immunol 32: 450-459.

Guiliano DB, Oksov Y, Lustigman S, Gounaris K, Selkirk ME 2009. Characterisation of novel protein families secreted by muscle stage larvae of Trichinella spiralis. Int J Parasitol 39: 515-524.

Khan WI, Blennerhasset PA, Varghese AK, Chowdhury SK, Omsted P, Deng Y, Collins SM 2002. Intestinal nematode infection ameliorates experimental colitis in mice. Infect Immun 70: 5931-5937.

Maizels RM, Balic A, Gomez-Escobar N, Nair M, Taylor MD, Allen JE 2004. Helminth parasites - masters of regulation. Immunol Rev 201: 89-116.

Maizels RM, Yazdanbakhsh M 2003. Regulation of the immune response by helminth parasites: cellular and molecular mechanisms. Nat Rev Immunol 3: 733-743.

Motomura Y, Wang H, Deng Y, El-Sharkawy RT, Verdu EF, Khan WI 2009. Helminth antigen-based strategy to ameliorate inflammation in an experimental model of colitis. Clin Exp Immunol 155: 88-95.

Nagano I, Wu Z, Takahashi Y 2009. Functional genes and proteins of Trichinella spp. Parasitol Res 104: 197-207.

Robert F, Weil B, Kassis N, Dupouy-Camet J 1996. Investigation of immunofluorescence cross-reactions against Trichinella spiralis by Western blot (immunoblot) analysis. Clin Diagn Lab Immunol 5: 575-577.

Romarís F, Escalante M, Lorenzo S, Bonay P, Gárate T, Leiro J, Ubeira FM 2002. Monoclonal antibodies raised in Btk(xid) mice 
reveal new antigenic relationships and molecular interactions among gp53 and other Trichinella glycoproteins. Mol Bioche Parasitol 125: 173-183.

Saunders KA, Raine T, Cooke A, Lawrence CE 2007. Inhibition of autoimmune type 1 diabetes by gastrointestinal helminth infection. Infect Immun 75: 397-407.

Wilson MS, Maizels RM 2004. Regulation of allergy and autoimmunity in helminth infection. Clin Rev Allergy Immunol 26: 35-50.

Wisnewski N, McNeil M, Grieve RB, Wassom DL 1993. Charac- terization of novel fucosyl- and tyvelosyl-containing glycoconjugates from Trichinella spiralis muscle stage larvae. Mol Biochem Parasitol 61: 25-35.

Wu Z, Nagano I, Nakada T, Takahashi Y 2002. Expression of excretory and secretory protein genes of Trichinella at muscle stage differs before and after cyst formation. Parasitol Int 51: 155-161.

Wu Z, Sofronic-Milosavljevic Lj, Nagano I, Takahashi Y 2008. Trichinella spiralis: nurse cell formation with emphasis on analogy to muscle cell repair. Parasite Vectors 19: 27. 